\title{
Polynuclear palladium complexes with biogenic polyamines: AFM and voltammetric characterization
}

\author{
O. Corduneanu ${ }^{\text {a }}$, A.-M. Chiorcea-Paquim ${ }^{\text {a }}$, S.M. Fiuza ${ }^{\text {b }}$, M.P.M. Marques ${ }^{\text {b }}$, A.M. Oliveira-Brett ${ }^{\text {a,* }}$ \\ a Departamento de Quimica, Faculdade de Ciências e Tecnologia, Universidade de Coimbra, Portugal \\ b Unidade IED "Ouímica-Física Molecular" E Departamento de Bioquímica, Faculdade de Ciências e Tecnologia, Universidade de Coimbra, Portugal
}

\section{A R T I C L E I N F O}

\section{Article history:}

Received 13 June 2009

Received in revised form 6 August 2009

Accepted 9 August 2009

Available online 18 August 2009

\section{Keywords:}

Biogenic polyamines

Polynuclear Pd(II) complexes

Voltammetry

AFM

\begin{abstract}
A B S T R A C T
Polynuclear Pd(II) complexes with biogenic polyamines present great potential clinical importance, due to their antiproliferative and cytotoxic activity coupled to less severe side-effects. The adsorption process and the redox behaviour of two polynuclear palladium chelates with spermine (Spm) and spermidine (Spd), $\mathrm{Pd}(\mathrm{II})-\mathrm{Spm}$ and Pd(II)-Spd, as well as of their ligands Spm and Spd, were studied using atomic force microscopy (AFM) and voltammetry at highly oriented pyrolytic graphite and glassy carbon electrodes. AFM revealed different adsorption patterns and degree of surface coverage, correlated with the chelate structure, concentration of the solution, applied potential and voltammetric behaviour of the Spm, Spd, Pd(II)-Spm and Pd(II)-Spd systems.

The voltammetric study of Spm and Spd showed that these biogenic polyamines undergo an irreversible and $\mathrm{pH}$-dependent oxidation. In acid medium the polyamines are fully protonated, rendering their oxidation more difficult. With increasing $\mathrm{pH}$ the oxidation potential for both Spm and Spd is shifted to less positive values, indicating a greater ease of oxidation in alkaline medium. The Pd(II)-Spm and Pd(II)-Spd complexes dissociate at high negative or high positive potentials. The application of a positive potential induced the oxidation of these Pd complexes and the formation of mixed layers of palladium oxides, Spm/Spd and Pd(II)-Spm/Pd(II)-Spd.
\end{abstract}

(C) 2009 Elsevier B.V. All rights reserved.

\section{Introduction}

The biogenic polyamines spermidine ( $\mathrm{Spd}, \mathrm{H}_{2} \mathrm{~N}\left(\mathrm{CH}_{2}\right)_{3} \mathrm{NH}\left(\mathrm{CH}_{2}\right)_{4} \mathrm{NH}_{2}$ ) and spermine (Spm, $\left.\mathrm{H}_{2} \mathrm{~N}\left(\mathrm{CH}_{2}\right)_{3} \mathrm{NH}\left(\mathrm{CH}_{2}\right)_{4} \mathrm{NH}\left(\mathrm{CH}_{2}\right)_{3} \mathrm{NH}_{2}\right)$ ) are organic bases comprising three and four atoms of nitrogen respectively, separated by linear alkyl chains which act as variable length linkers between the amine units [1]. Scheme 1 shows the structures of these polyamines as dications (physiological form). However, several conformers are possible at room temperature, due to the presence of multiple ionisation sites and the occurrence of intramolecular H-bonds (namely $\mathrm{NH} \cdots \mathrm{N}$ ) [2]. These compounds are present in all eukaryotic organisms and are essential for cell proliferation and differentiation (both in normal and malignant cells) $[3,4]$. Owing to their polycationic nature, polyamines influence the conformation of many biological macromolecules (e.g. nucleic acids), through electrostatic interactions with specific anionic sites such as the negatively charged phosphate groups of DNA $[5,6]$.

With the aim of finding novel antineoplastic therapeutic strategies, polyamines have been considered for the development of new anticancer drugs, having been used as polydentate ligands in polynuclear metal

\footnotetext{
* Corresponding author. Departamento de Química, Faculdade de Ciências e Tecnologia, Universidade de Coimbra, 3004-535 Coimbra, Portugal. Tel./fax: +351239835295.

E-mail address: brett@ci.uc.pt (A.M. Oliveira-Brett).
}

chelates, a third generation class of anticancer agents with specific chemical and biological properties [7-16]. In fact, the presence of more than one metal centre in these complexes allows them to bind more effectively to DNA, being considered as promising alternatives to cisplatin (cis-dichlorodiammineplatinum(II), cis- $\mathrm{Pt}\left(\mathrm{Cl}_{2}\left(\mathrm{NH}_{3}\right)_{2}\right)$ that displays a well known anticancer activity but also deleterious toxicity and development of resistance [16-18]. In polynuclear polyamine complexes, the flexible linear amine ligands, particularly Spm and Spd, may form a molecular scaffolding due to the presence of the cationic groups located at regular intervals within the molecule, thus enabling a selective, nonconventional, interaction with the electronegative groups of DNA.

In a rational approach for the development of improved antineoplastic agents, not only the ligands but also the metal centres have been varied. Substitution of platinum by palladium, for instance, has been one of the strategies reported in a number of studies $[7,8,12,19-22]$. The first results obtained for Pd(II) complexes have evidenced a quite low antitumour activity, attributed to the high lability of the $\mathrm{Pd}(\mathrm{II})$ centre (as compared to the analogous $\mathrm{Pt}(\mathrm{II})$ complexes [23]) and possible deactivation by reaction with biomolecules other than DNA (mainly S-containing compounds), which can result in a quite reduced bioavailability of the drug at the molecular target and a consequent decreased activity. However, the increasing use of chelating ligands (e.g. N-containing donors) has been shown to lead to a marked decrease of the Pd(II) complex lability [7,8,24,25], as 

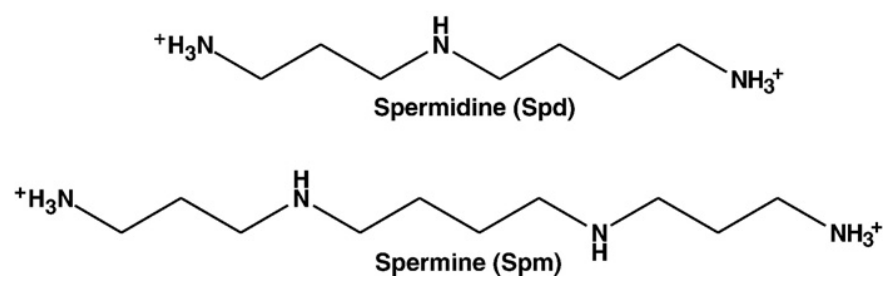

Scheme 1. Molecular structures of the polyamines spermidine (Spd) and spermine (Spm). (N-protonated species, predominant at physiological conditions).

well as to a shift of the cis-trans equilibrium towards the formation of the cis isomer. Furthermore, the use of ligands with significant biological activity and affinity for DNA may also provide specific and improved antitumour activity, sometimes even higher than that measured for cisplatin $[7,26]$. Several cationic polynuclear Pd(II) chelates comprising two or three cisplatin-like moieties linked by variable length aliphatic polyamines, have recently been assessed as to their antiproliferative and cytotoxic effects towards several human cancer cell lines [11,13,14,21], and this activity has been related to their conformational preferences at physiological conditions $[27,28]$. In fact, the understanding of the mechanisms of action of these metal-based agents is essential for the establishment of structure-activity relationships that will enable a rational design of new and improved anticancer drugs.

The present paper is the first report of a voltammetric and atomic force microscopy (AFM) study of two polynuclear Pd(II) complexes, with Spm and Spd as polydentate ligands. $\mathrm{Pd}(\mathrm{II})-\mathrm{Spm}$ is a dinuclear $2,2 / \mathrm{c}, \mathrm{c}$ chelate of general formula $\left(\mathrm{PdCl}_{2}\right)_{2} \mathrm{Spm}$, that contains one tetraamine ligand (Spm) acting as a linker for both metal centres, yielding two identical six-membered intramolecular rings in a trans orientation relative to each other. $\mathrm{Pd}(\mathrm{II})-\mathrm{Spd}$ is a trinuclear 2,2,2/c,c,c $\mathrm{Pd}(\mathrm{II})$ chelate of general formula $\left(\mathrm{PdCl}_{2}\right)_{3}(\mathrm{Spd})_{2}$, comprising two Spd units as bridging ligands between the three metal centres.

The process of adsorption of Pd(II)-Spm and Pd(II)-Spd, as well as of their ligands Spm and Spd, was studied on a highly oriented pyrolytic graphite (HOPG) electrode using magnetic AC mode atomic force microscopy (MAC Mode AFM). Direct electrochemical oxidation of the biogenic polyamines Spm and Spd at a glassy carbon (GC) electrode has also been performed without the use of mediators, by cyclic voltammetry (CV), differential pulse (DP) and square wave (SW) voltammetry over a wide range of $\mathrm{pH}$.

\section{Experimental}

\subsection{Materials and reagents}

Spermidine and spermine were purchased from Sigma-Aldrich and kept at $4{ }^{\circ} \mathrm{C}$. Solutions of either Spm or Spd were freshly prepared before each experiment by dilution of the appropriate quantity in the supporting electrolyte. The supporting electrolyte solutions used were: $\mathrm{pH} 4.5$ and $5.10 .1 \mathrm{M}$ acetate buffer, $\mathrm{pH} 5.9,7.0$ and $8.00 .1 \mathrm{M}$ phosphate buffer and $\mathrm{pH} 8.5,9.8$ and $11.10 .1 \mathrm{M}$ ammonium buffer. Pd-spermine ( $\mathrm{Pd}(\mathrm{II})-\mathrm{Spm}$ or $\left.\left(\mathrm{PdCl}_{2}\right)_{2} \mathrm{Sp}\right)$ and Pd-spermidine $\left(\mathrm{Pd}(\mathrm{II})-\mathrm{Spd}\right.$ or $\left.\left(\mathrm{PdCl}_{2}\right)_{3}(\mathrm{Spm})_{2}\right)$ were synthesised in the Research Unit "Molecular Physical-Chemistry", Coimbra, Portugal, according to published procedures $[9,10]$, with slight modifications. Solutions of either Pd(II)-Spm or Pd(II)-Spd were freshly prepared before each experiment by dilution of the appropriate quantity in $\mathrm{pH} 7.00 .1 \mathrm{M}$ phosphate buffer. All solutions were prepared using analytical grade reagents and purified water from a Millipore Milli- $Q$ system (conductivity $\leq 0.1 \mu \mathrm{S} \mathrm{cm}^{-1}$ ).

Nitrogen saturated solutions were obtained by bubbling high purity $\mathrm{N}_{2}$ for a minimum of $10 \mathrm{~min}$ through the solution and a continuous flow of pure gas was maintained over the solution during the voltammetric experiments.
Microvolumes were measured using EP-10 and EP-100 Plus Motorized Microliter Pippettes (Rainin Instrument Co. Inc., Woburn, USA). The pH measurements were carried out with a Crison micropH $2001 \mathrm{pH}-$ meter with an Ingold combined glass electrode. All experiments were done at room temperature $\left(25 \pm 1{ }^{\circ} \mathrm{C}\right)$.

\subsection{Atomic force microscopy}

HOPG, grade ZYB of $15 \times 15 \times 2 \mathrm{~mm}^{3}$ dimensions, from Advanced Ceramics Co., USA, was used as a substrate in the MAC Mode AFM study. The HOPG was freshly cleaved with adhesive tape prior to each experiment and imaged by AFM in order to establish its cleanliness.

AFM was performed with a PicoSPM controlled by a MAC mode module and interfaced with a PicoScan controller from Agilent Technologies, Tempe, AZ, USA. All the AFM experiments were performed with a CS AFM S scanner with a scan range of $6 \mu \mathrm{m}$ in $x-y$ and $2 \mu \mathrm{m}$ in $z$, from Agilent Technologies. Silicon type II MAClevers of $225 \mu \mathrm{m}$ length, $2.8 \mathrm{~N} \mathrm{~m}^{-1}$ spring constants and $60-90 \mathrm{kHz}$ resonant frequencies in air (Agilent Technologies) were used. All AFM images were topographical and were taken with 256 samples/line $\times 256$ lines and scan rates of 0.8-2.0 lines $\mathrm{s}^{-1}$. When necessary, MAC Mode AFM images were processed by flattening in order to remove the background slope and the contrast and brightness were adjusted. Section analyses were performed with PicoScan software version 5.3.3, Agilent Technologies, and with Origin version 6.0, Microcal Software, Inc., USA.

\subsection{Voltammetric parameters and electrochemical cells}

The voltammetric experiments were performed using an Autolab running with GPES 4.9 software, Eco-Chemie, Utrecht, The Netherlands. CVs were recorded at scan rates of 50 and $100 \mathrm{mVs}^{-1}$. The DP voltammetry conditions were: pulse amplitude of $50 \mathrm{mV}$, pulse width of $70 \mathrm{~ms}$, step potential of $2 \mathrm{mV}$ and scan rate of $5 \mathrm{mVs}^{-1}$. SW voltammetry conditions were: frequency of $8 \mathrm{~Hz}$, amplitude of $50 \mathrm{mV}$ and a potential increment of $2 \mathrm{mV}$ (effective scan rate of $15 \mathrm{mV} \mathrm{s}^{-1}$ ).

Measurements were carried out in a $0.5 \mathrm{~mL}$ one-compartment electrochemical cell using a GC electrode $(d=1.5 \mathrm{~mm})$, with a Pt wire counter electrode, and an $\mathrm{Ag} / \mathrm{AgCl}(3 \mathrm{M} \mathrm{KCl})$ electrode as reference.

\subsection{Sample preparation}

The adsorption of Spm, Spd, Pd(II)-Spm and Pd(II)-Spd onto HOPG and GC electrodes was studied by MAC mode AFM in air and voltammetric methods, using the procedures described below.

Procedure 1 - Spm, Spd, Pd(II)-Spm and Pd(II)-Spd modified HOPG. The Spm, Spd, Pd(II)-Spm and Pd(II)-Spd modified HOPG were obtained by spontaneous adsorption, depositing $200 \mu \mathrm{L}$ samples of the desired Spm, Spd, Pd(II)-Spm or Pd(II)-Spd solution onto freshly cleaved HOPG surfaces and incubating for $10 \mathrm{~min}$. The excess of solution was removed with Millipore Milli-Q water and the electrode was dried in a sterile atmosphere.

Procedure 2 - Electrodeposited Pd(II)-Spm and Pd(II)-Spd modified HOPG. The electrodeposition of Pd(II)-Spm and $\mathrm{Pd}(\mathrm{II})-\mathrm{Spd}$ was performed from concentrations of $10 \mu \mathrm{M}$ Pd(II)-Spm and Pd(II)-Spd in in pH 7.0 0.1 M phosphate buffer solutions, by applying $+1.2 \mathrm{~V}$ vs. $\mathrm{Ag} / \mathrm{AgCl}(3 \mathrm{M} \mathrm{KCl})$, during $30 \mathrm{~min}$. The excess of solution was removed with Millipore Milli-Q water and the electrode was dried in a sterile atmosphere.

Procedure 2 - Electrodeposited Pd(II)-Spm and Pd(II)-Spd modified GC electrode. The electrodeposition of palladium complexes was performed from solutions of $2 \mathrm{mM}$ $\mathrm{Pd}(\mathrm{II})-\mathrm{Spm}$ and $\mathrm{Pd}(\mathrm{II})-$ Spd in $\mathrm{pH} 7.00 .1 \mathrm{M}$ phosphate buffer, by applying $+1.2 \mathrm{~V}$ vs. $\mathrm{Ag} / \mathrm{AgCl}$ 
( $3 \mathrm{M} \mathrm{KCl}$ ), during $30 \mathrm{~min}$. After electrodeposition the electrode was washed with Millipore Milli-Q water and transferred to $\mathrm{pH} 4.50 .1 \mathrm{M}$ acetate buffer.

\section{Results and discussion}

The Spm, Spd, Pd(II)-Spm and Pd(II)-Spd adsorption and the redox processes were studied using AFM and voltammetry. The results obtained for the biogenic polyamines Spm and Spd are essential to explain the voltammetric behaviour and the interaction established by the polynuclear palladium complexes Pd(II)-Spm and Pd(II)-Spd with the GC and HOPG electrodes.

The AFM study of Spm, Spd, Pd(II)-Spm and Pd(II)-Spd was performed using the procedures from Section 2.4. As described below, the topographical images clearly showed the different ability of Spm, Spd, Pd(II)-Spm and Pd(II)-Spd to interact and adsorb spontaneously on carbon electrode surfaces, forming different morphological structures and films. For the correct evaluation of the adsorbed molecules and films, the HOPG was used as substrate, which presents a root-mean-square roughness (r.m.s) of less than $0.06 \mathrm{~nm}$ for a $1000 \times 1000 \mathrm{~nm}^{2}$ surface area. The GC electrode used for the voltammetric characterization was much rougher, with $2.10 \mathrm{~nm}$ r.m. s. roughness for the same surface area, therefore unsuitable for AFM surface characterization. Furthermore, the experiments using GC and HOPG electrodes showed similar electrochemical behaviour.

\subsection{Adsorption process and redox behaviour of spermine and spermidine}

\subsubsection{AFM characterization of adsorbed Spm and Spd}

The spontaneous adsorption onto HOPG of Spm and Spd, ligands in the $\mathrm{Pd}(\mathrm{II})-\mathrm{Spm}$ and $\mathrm{Pd}(\mathrm{II})-\mathrm{Spd}$ complexes, was performed using Procedure 1 from Section 2.4 .
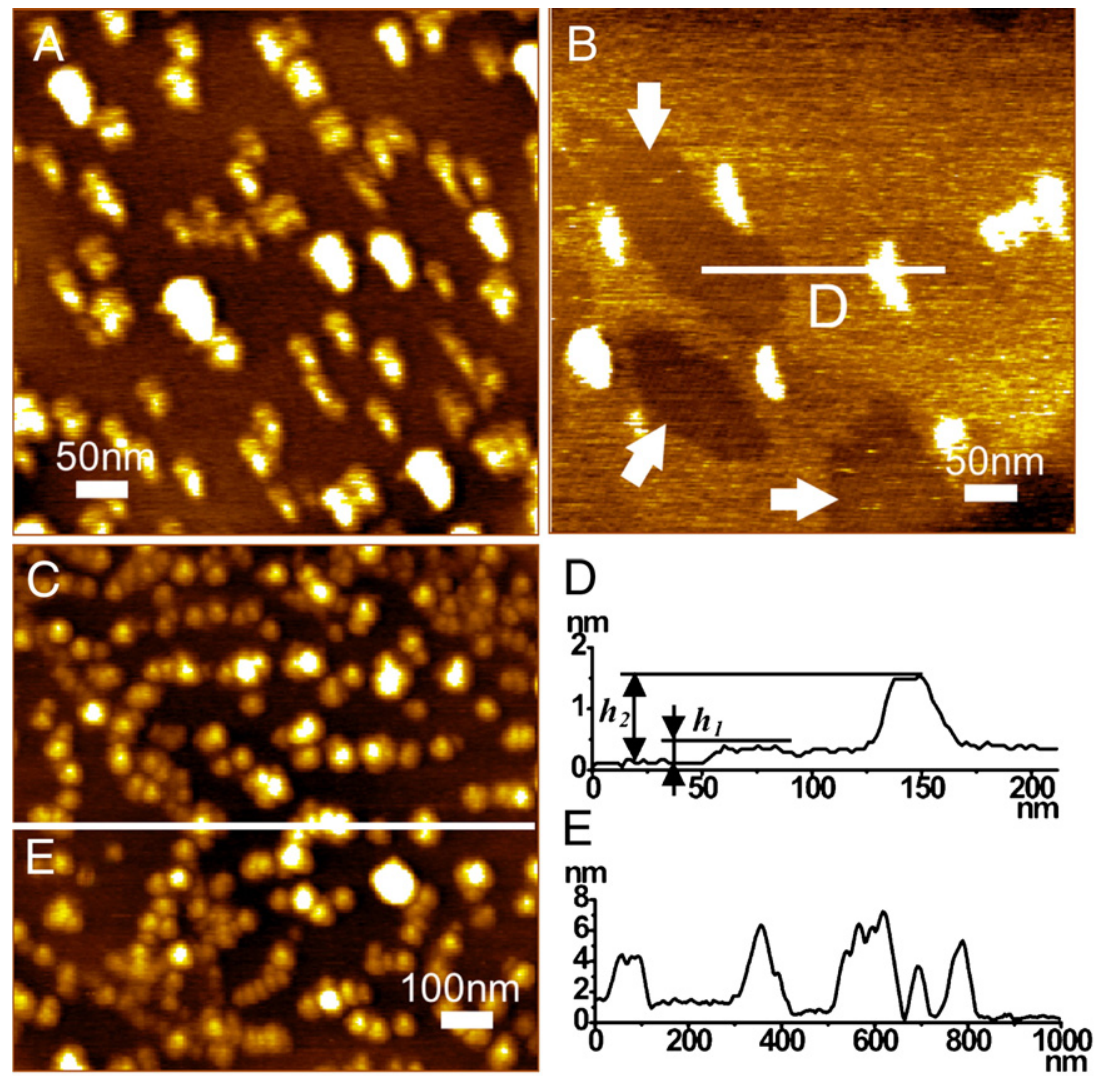

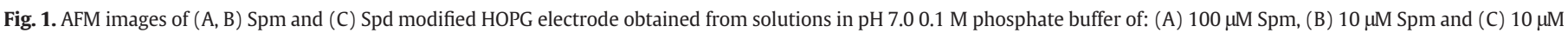
Spd. (D, E) Cross section profiles through the white lines in the images (B) and (C).
AFM images in air of the Spm modified HOPG surface obtained from a concentrated solution of $100 \mu \mathrm{M}$ Spm, Fig. 1A, showed spherical aggregates assembled on the HOPG surface with $2.5 \pm 0.4 \mathrm{~nm}$ height. Larger aggregates and molecular clusters up to $5.0 \mathrm{~nm}$ of height could be also observed in the images. The number of aggregates decreased with decreasing concentrations down to $50 \mu \mathrm{M}$ (data not shown). For solutions of $10 \mu \mathrm{M} \mathrm{Spm}$, Fig. 1B, the AFM images showed the formation of a thin Spm film with large pores (pointed out by red arrows in the image from Fig. 1B) that exhibit the HOPG surface underneath. Performing section analysis on the thin film, a mean height and standard deviation of $0.4 \pm 0.10 \mathrm{~nm}$ was measured, $h_{1}$ from Fig. 1D, which corresponds to a monolayer formed by hydrophobic interactions between Spm alkyl chains and the hydrophobic HOPG surface. In addition, spherical aggregates and clusters with non-uniform heights between 1.0 and $5.0 \mathrm{~nm}, h_{2}$ from Fig. 1D, were embedded into the Spm monolayer. The formation of the Spm monolayer observed in the AFM images and the decrease of the number of molecular clusters with decreasing Spm concentration demonstrated that the uniformly distributed Spm aggregates are not an artefact, but are related to the adsorption onto HOPG.

As expected, the AFM images of a Spd modified HOPG surface obtained from a solution of $50 \mu \mathrm{M}$ (data not shown) and $10 \mu \mathrm{M}$ Spd, Fig. 1C, showed a similar pattern of adsorption as obtained for the Spm molecules, with $4.1 \pm 1.0 \mathrm{~nm}$ height spherical aggregates, Fig. 1E.

\subsubsection{Voltammetric characterization of Spm and Spd}

The voltammetric behaviour of the biogenic polyamines Spm and Spd was studied initially by cyclic voltammetry in solutions of $100 \mu \mathrm{M}$ in $\mathrm{pH} 7.00 .1 \mathrm{M}$ phosphate buffer saturated with $\mathrm{N}_{2}$. Successive scans were obtained in the solution of Spm and Spd, starting at $E_{\mathrm{i}}=0.00 \mathrm{~V}$ in a potential range of $-1.00 \mathrm{~V}$ to $+1.40 \mathrm{~V}$ with a scan rate of $\nu=100 \mathrm{mV} \mathrm{s}^{-1}$. The CVs showed a single, irreversible oxidation peak 
at high positive potentials, well defined in the case of Spm, Fig. 2A-B. A decrease of the peak current with the number of scans recorded, followed by a stabilization of the peak current, and the absence of other peaks related to the formation of electroactive oxidation products, was also observed. After five scans in solution, the GC electrode was rinsed and transferred to the electrolyte medium of $\mathrm{pH}$ 7.0, where CVs were recorded in the same potential range. The presence of Spm and Spd adsorbed on GC electrode surface was observed only in the first scan (not shown), meaning that the adsorbed aggregates and films were not strongly attached to the electrode surface, being desorbed during just one CV.

The SW voltammograms, Fig. 2A-B Inserts, showed similar results to the $\mathrm{CV}$ s, i.e. the presence of a single, irreversible oxidation peak for both Spm and Spd. The voltammograms were recorded at a frequency of $8 \mathrm{~Hz}$ (effective scan rate of $15 \mathrm{mVs}^{-1}$ ) showing a well defined oxidation peak over the whole $\mathrm{pH}$ range studied, when compared with the CV results recorded at a scan rate of $100 \mathrm{mVs}^{-1}$, which suggests a slow oxidation kinetics.

The influence of $\mathrm{pH}$ on the oxidation peak potential of Spm and Spd was studied by DP voltammetry in different supporting electrolytes with the ionic strength $0.1 \mathrm{M}$. The effect of the $\mathrm{pH}$ on the oxidation potential and on the peak currents obtained is shown in Fig. 3A for Spm and in Fig. 3B for Spd. The DP voltammograms showed that the oxidation peak potential was shifted to less positive values as the $\mathrm{pH}$ increases, indicating greater ease of oxidation in alkaline medium.
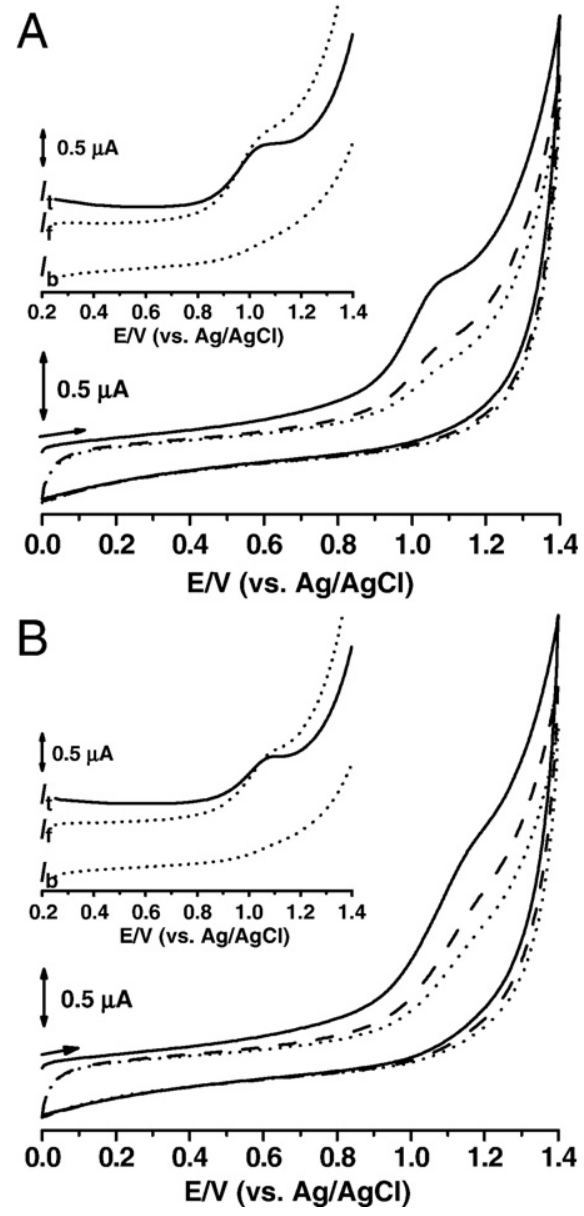

Fig. 2. CVs in pH 7.0 0.1 M phosphate buffer of $100 \mu \mathrm{M}$ : (A) Spm and (B) Spd: (-) 1st $(\cdots) 2$ nd and $(\cdots) 5$ th scan. Inserts SW voltammograms: $I_{\mathrm{t}}-$ total, $I_{\mathrm{t}}-$ forward and $I_{\mathrm{b}}-$ backward currents.
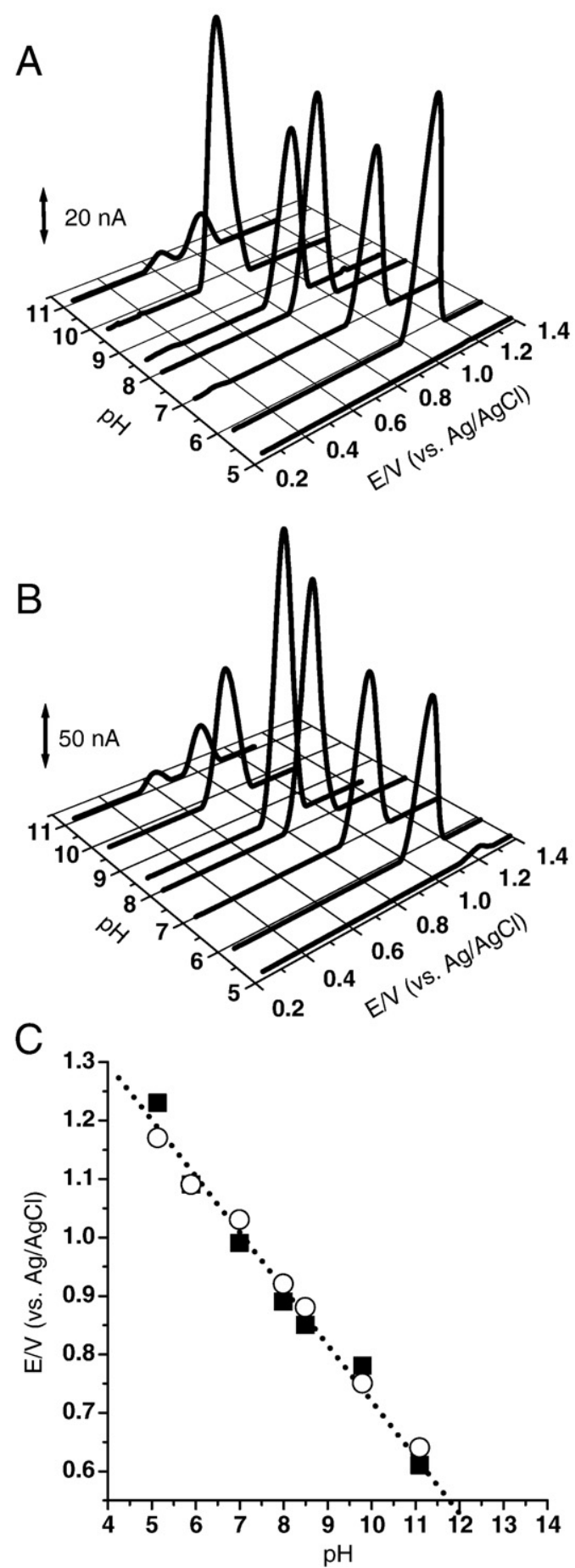

Fig. 3. DP voltammograms: (A, B) 3D plot in different supporting electrolytes as a function of pH of $100 \mu \mathrm{M}$ (A) Spm and (B) Spd; (C) Plot of $E_{\mathrm{pa}}$ Vs. pH for (匹) Spm and (O) Spd. Slope of the dotted line $93 \mathrm{mV}$ per $\mathrm{pH}$ unit.

It was also observed that at $\mathrm{pH}$ 11.1 $0.1 \mathrm{M}$ ammonium buffer two oxidation peaks for each of the polyamines were detected, with low currents in relation to the other supporting electrolytes. At pH 5.1 $0.1 \mathrm{M}$ acetate buffer no oxidation peak was observed in the case of Spd, whereas for Spm a small oxidation peak at $E_{\mathrm{pa}}=+1.23 \mathrm{~V}$ was detected with a low current of only $6 \mathrm{nA}$.

In supporting electrolytes with $\mathrm{pH} \leq 5.1$ no charge transfer reaction was detected. The significant increase in the oxidation potential and the decrease of peak currents in acid medium showed a 
low capacity of these amines for electro-oxidation, which can be explained considering their protonation reactions. The protonated form is more deficient in electrons than the neutral, not protonated form, thus reducing the oxidation capacity. The results indicate that in acid medium the polyamines are fully protonated and that they hardly suffer oxidation at pH 5.1, as observed by the high oxidation potential and low peak currents.

The number of electrons involved in the oxidation process was calculated from the width at half height $W_{1 / 2}$ of the oxidation peak, where $W_{1 / 2}=110 \mathrm{mV}$ in the case of Spd, and $W_{1 / 2}=90 \mathrm{mV}$ for Spm, thus for both polyamines until $\mathrm{pH} 11.1$ the oxidation occurs with the transfer of one electron. Representing $E_{\mathrm{p}} v s . \mathrm{pH}$, a linear dependence of the oxidation peak with the $\mathrm{pH}$ in the studied range was observed, Fig. 3C, with a slope of $89 \mathrm{mV}$ per $\mathrm{pH}$ unit for Spd and of $93 \mathrm{mV}$ per $\mathrm{pH}$ unit for Spm. These slopes indicate that in the oxidation process for Spm and Spd the transfer of protons also takes place. In the oxidation mechanism more protons than electrons are involved.

Comparing the oxidation potential of Spm and Spd, very similar values were found which indicates the same oxidation mechanism. In vivo there is an active interconversion of Spm and Spd, and between Spd and putrescine $\left(\mathrm{H}_{2} \mathrm{~N}\left(\mathrm{CH}_{2}\right)_{4} \mathrm{NH}_{2}\right)$, mediated by oxidase enzymes. In this cycle of catabolic interconversion, Spm is degraded in an oxidative manner to Spd and from Spd to putrescine. The first step in this sequence [3] is the acetylation of terminal amino groups:

$\mathrm{R}-\mathrm{CH}_{2}-\mathrm{NH}_{3}^{+} \stackrel{\text { Enzyme }}{\longrightarrow} \mathrm{RCHO}$

The second step is the oxidative separation of the acetylated polyamines [3], with the removal of the aminopropyl residues [4]:

$\mathrm{R}_{1}-\mathrm{CH}_{2}-\mathrm{NH}-\mathrm{CH}_{2}-\mathrm{R}_{2}+\mathrm{O}_{2}+\mathrm{H}_{2} \mathrm{O} \rightarrow \mathrm{R}_{1}-\mathrm{CHO}+\mathrm{H}_{2} \mathrm{~N}-\mathrm{CH}_{2}-\mathrm{R}_{2}+\mathrm{H}_{2} \mathrm{O}$
In this case, a possible general reaction [29] at the glassy carbon electrode is:

$\mathrm{R}-\mathrm{CH}_{2}-\mathrm{NH}_{2}+2 \mathrm{OH}^{-} \rightarrow \mathrm{R}-\mathrm{CHO}+\mathrm{NH}_{3}+\mathrm{H}_{2} \mathrm{O}+2 \mathrm{e}^{-}$

With the oxidative deamination of the primary amino groups, each of the interconversion intermediaries can be transformed into aldehydes, which in vivo may still suffer oxidation to an amino acid or a gamma-lactam. The end products of the catabolism and of polyamine acetylation are then subjected to urinary excretion [4]

\subsection{Adsorption process and redox behaviour of polynuclear palladium} chelates with Spm and Spd

\subsubsection{AFM characterization of spontaneously adsorbed Pd(II)-Spm and $\operatorname{Pd}($ II $)-S p d$}

The capacity of Pd(II)-Spm and Pd(II)-Spd to interact and adsorb on carbon electrode surfaces forming different morphological films was first investigated using AFM in air. The Pd(II)-Spm and Pd(II)-Spd modified HOPG electrodes were obtained by spontaneous adsorption during $10 \mathrm{~min}$, from different concentrations of Pd(II)-Spm and Pd(II)-Spd, using the method described in Procedure 1.

AFM images of the Pd(II)-Spm modified HOPG obtained for a $50 \mu \mathrm{M}$ $\mathrm{Pd}(\mathrm{II})-\mathrm{Spm}$ solution showed aggregates and clusters of $2.4 \pm 0.6 \mathrm{~nm}$ height, Fig. 4A. Decreasing the concentration to $10 \mu \mathrm{M}$, Fig. 4B and C, the distribution and height of the molecular aggregates and clusters decreased to $1.3 \pm 0.2 \mathrm{~nm}$, and showed a tendency to attach preferentially near the defects on the HOPG step edges.

AFM images of the Pd(II)-Spd modified HOPG obtained from concentrations of $50 \mu \mathrm{M} \mathrm{Pd}(\mathrm{II})-\mathrm{Spd}$ solution, Fig. 5A, showed the
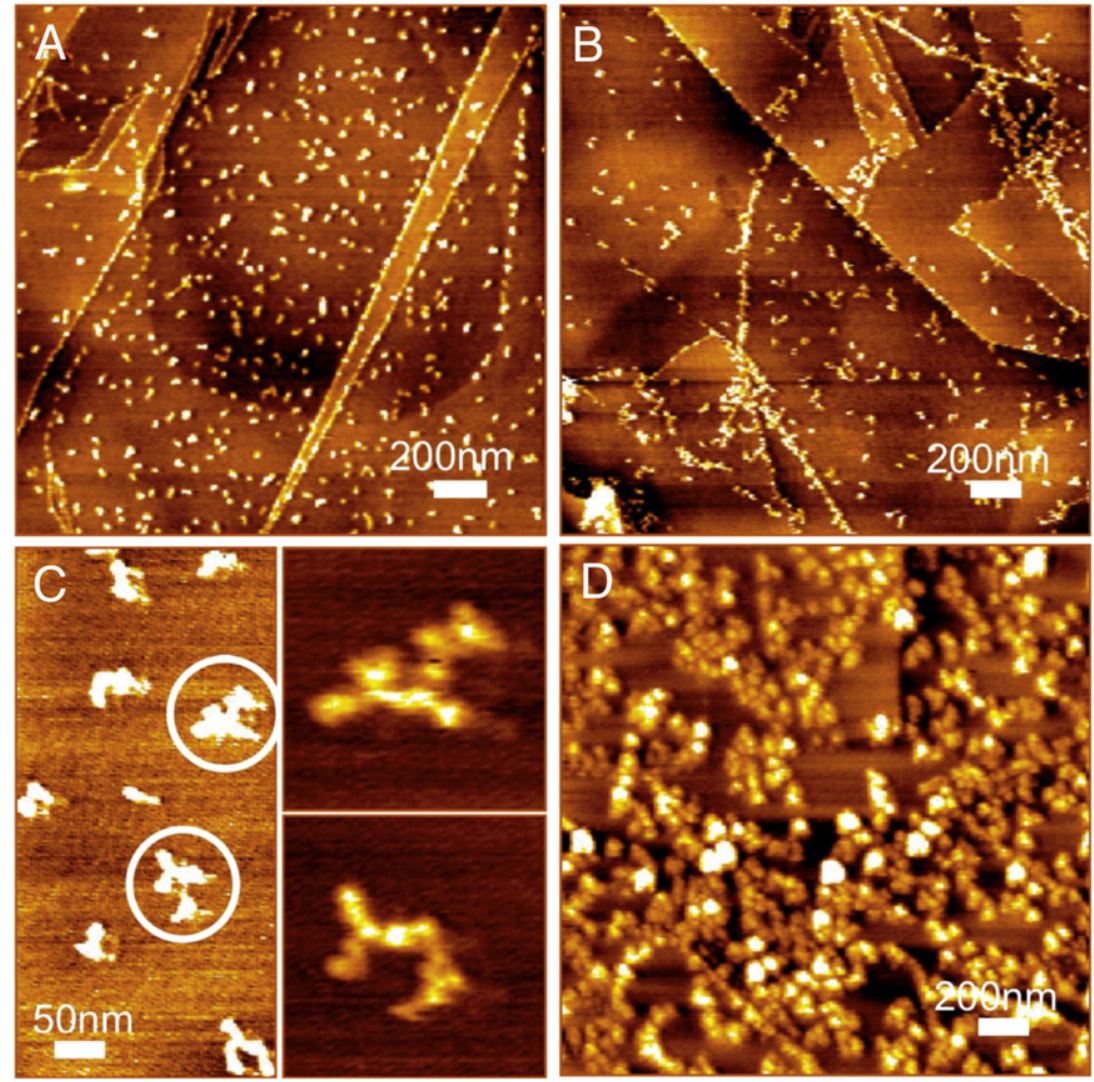

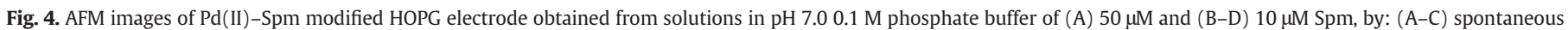
adsorption during $10 \mathrm{~min}$ and (D) electrodeposition at $+1.20 \mathrm{~V}$, vs. $\mathrm{Ag} / \mathrm{AgCl}$, during $30 \mathrm{~min}$. 

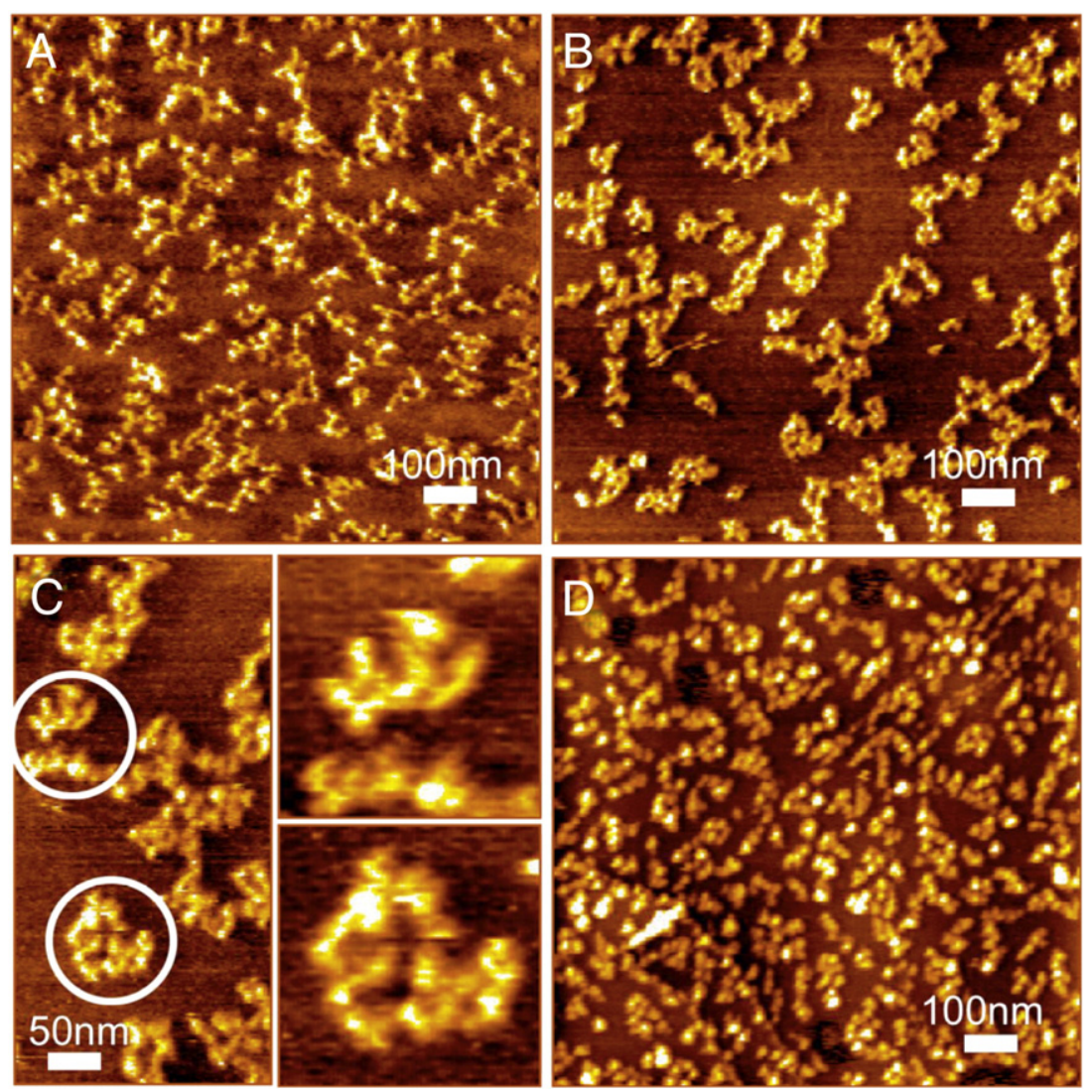

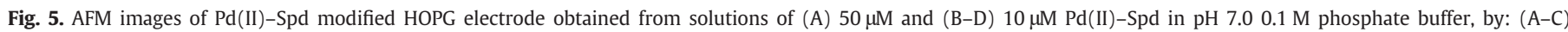
spontaneous adsorption during $10 \mathrm{~min}$ and (D) electrodeposition at $+1.20 \mathrm{~V}$, vs. $\mathrm{Ag} / \mathrm{AgCl}$, during $30 \mathrm{~min}$.

formation of $2.0 \pm 0.3 \mathrm{~nm}$ height molecular clusters, slightly decreasing to $1.7 \pm 0.2 \mathrm{~nm}$ height at $10 \mu \mathrm{M}$ Pd(II)-Spd, Fig. 5B and C.

The Pd(II)-Spd complexes demonstrated an increased adsorption onto HOPG, when compared with Pd(II)-Spm, due to the existence of a larger number of palladium atoms and of ligand molecules in its molecular structure. Indeed Pd(II)-Spd has three palladium atoms, which increase the spontaneous adsorption onto HOPG [30,31], and two Spd molecules, vs. two palladium and one Spm in the case of $\mathrm{Pd}(\mathrm{II})-\mathrm{Spm}$. Additionally the larger Pd(II)-Spd complexes lead to the formation of larger and higher molecular clusters, Fig. 4C, compared with Pd(II)-Spm, Fig. 5C.

\subsubsection{Voltammetric characterization of Pd(II)-Spm and Pd(II)-Spd}

The voltammetric characterization of the complexes $\mathrm{Pd}(\mathrm{II})-\mathrm{Spm}$ and $\mathrm{Pd}(\mathrm{II})-\mathrm{Spd}$ at the GC electrode was first studied by CV in concentrated solutions of $2 \mathrm{mM}$ in pH 7.0 0.1 M phosphate buffer, saturated with $\mathrm{N}_{2}$. In Fig. 6 are shown successive CVs starting at $E_{\mathrm{i}}=0.00 \mathrm{~V}$ in the positive direction until $E_{1}=+1.40 \mathrm{~V}$ then going in the negative direction to progressively more negative applied potential limits. The CVs recorded showed that when the negative applied potential reaches $E_{2}=-0.50 \mathrm{~V}$ a complex electrochemical behaviour is observed, characteristic of the electrodeposition of palladium at the surface of the electrode $[30,31]$.

Continuing with the application of progressively more negative potential limits up to $E_{2}=-1.00 \mathrm{~V}$, other peaks and a large increase in peak currents were recorded. All peaks are related to different states of oxidation of the $\operatorname{Pd}(0)$ nanostructures electrodeposited at the surface of the electrode as the applied potential reaches sufficiently negative values. Once electrodeposited on the electrode, the $\operatorname{Pd}(0)$ nanostructures undergo several redox reactions, such as hydrogen evolution, palladium oxide formation and their reduction [30,31].
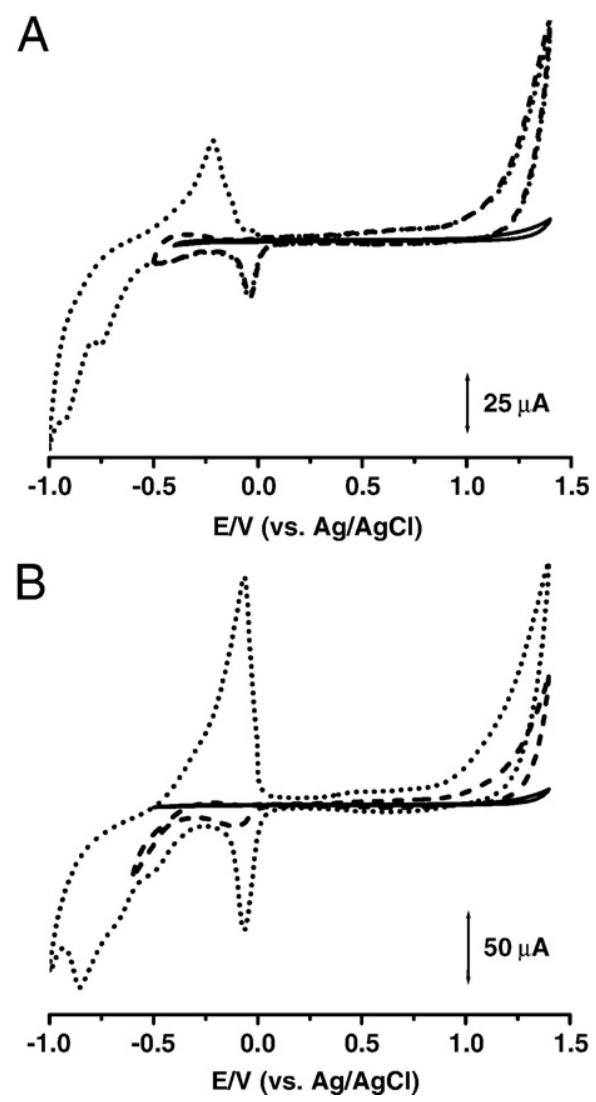

Fig. 6. CVs in pH 7.0 0.1 M phosphate buffer of $2 \mathrm{mM}(\mathrm{A}) \mathrm{Pd}(\mathrm{II})-\mathrm{Spm}$ and (B) Pd(II)-Spd: (一) 3rd, (..) 5th and ( $\bullet \cdot)$ 10th scan. Scan rate $100 \mathrm{mV} \mathrm{s}^{-1}$. 


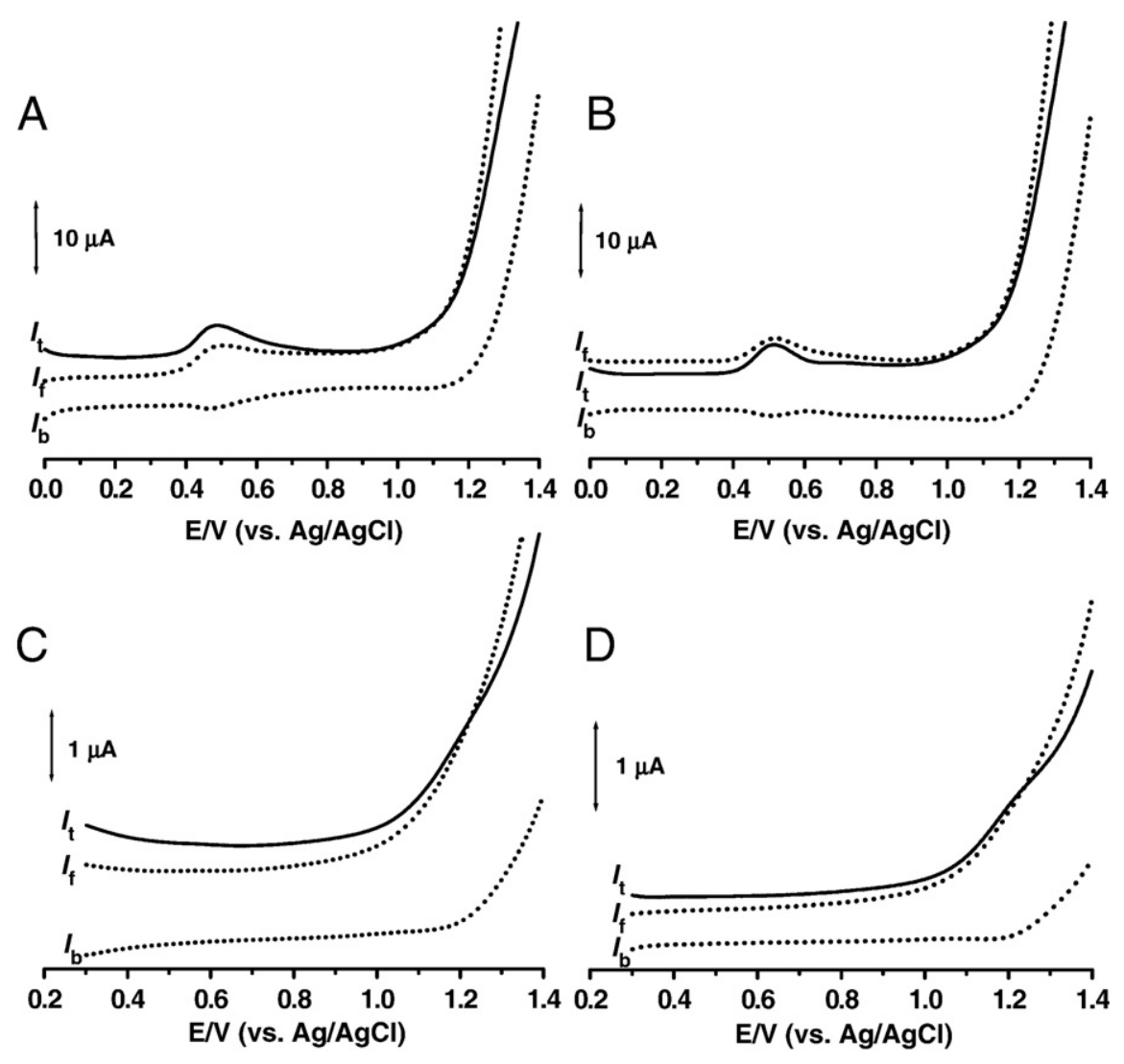

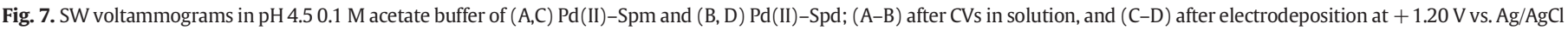
during 30 min from $2 \mathrm{mM}$ solutions in pH 7.0 0.1 M phosphate buffer, $I_{\mathrm{t}}-$ total, $I_{\mathrm{t}}-$ forward and $I_{\mathrm{b}}-$ backward currents. Frequency $8 \mathrm{~Hz}$.

After the CV scans in the $2 \mathrm{mM}$ solutions of either Pd(II)-Spm or Pd(II)-Spd, the electrode was washed and transferred to $\mathrm{pH} 4.5$ $0.1 \mathrm{M}$ acetate buffer. The presence of palladium oxides on the surface of the electrode was confirmed by SW voltammetry in supporting electrolyte $\mathrm{pH} 4.5$, Fig. 7A-B, which showed reversible oxidation processes at $+0.51 \mathrm{~V}$ and $+1.20 \mathrm{~V}$. It was also observed that in the case of the Pd(II)-Spd complex, the peak currents were higher in relation to those recorded for the Pd(II)-Spm complex, related to both the presence of more palladium centres in the $\mathrm{Pd}(\mathrm{II})-\mathrm{Spd}$ and a greater adsorption onto the carbon electrode surface of Pd(II)-Spd, Fig. 5A-C, when compared with Pd(II)-Spm, Fig. 4A-C.

Since in the experiments in solution $\operatorname{Pd}(0)$ was electrodeposited by applying a sufficiently negative potential, the electrodeposition at a high positive potential was also investigated in order to confirm that palladium could be removed from these complexes and deposited directly as palladium oxide.

\subsubsection{Electrodeposition of Pd(II)-Spm and Pd(II)-Spd}

To test the stability of Pd(II)-Spm and Pd (II)-Spd complexes for the removal and deposition of palladium on the surface of the electrode as palladium oxide, a potential of $E_{\text {dep }}=+1.20 \mathrm{~V}$ was applied during $30 \mathrm{~min}$, as described in Section 2.4, Procedures 2 and 3 and the electrodeposited Pd(II)-Spm and Pd(II)-Spd modified HOPG and GC electrodes were investigated by AFM and voltammetry, respectively.

The electrodeposited Pd(II)-Spm and Pd(II)-Spd modified HOPG surfaces were prepared in $10 \mu \mathrm{M}$ Pd(II)-Spm or Pd(II)-Spd, as described in Procedure 2. AFM images of electrodeposited Pd(II)-Spm, Fig. 4D, and $\mathrm{Pd}(\mathrm{II})-\mathrm{Spd}$, Fig. 5D, modified HOPG both showed the formation of a large number of molecular clusters on the surfaces of HOPG, that of $\mathrm{Pd}(\mathrm{II})-\mathrm{Spm}$ presented very non-uniform heights between $1.2 \mathrm{~nm}$ and $7.0 \mathrm{~nm}$, and more uniform, of $1.5 \pm 0.3 \mathrm{~nm}$, in the case of Pd(II)-Spd. The application of a positive potential induced the oxidation of the Pd-Spm complexes and the formation of a mixed multilayer of palladium oxides [30], Spm/Spd, and $\mathrm{Pd}(\mathrm{II})-\mathrm{Spm} / \mathrm{Pd}(\mathrm{II})-\mathrm{Spd}$ complexes on HOPG.

The electrodeposited Pd(II)-Spm and Pd(II)-Spd modified GCE, obtained from solutions of $2 \mathrm{mM}$ Pd(II)-Spm and Pd(II)-Spd, showed no charge transfer reactions in the first $\mathrm{CV}$ recorded in the positive direction in supporting electrolyte, Fig. $8(-)$ and zoom. Only after reversing the scan direction at $-1.00 \mathrm{~V}$ was the characteristic peak for hydrogen evolution observed, which means that while the palladium oxides were not detected at the scan rate of $50 \mathrm{mV} \mathrm{s}^{-1}$, their reduction in the negative direction of the scan leads to the formation of $\operatorname{Pd}(0)$ that can then adsorb and absorb hydrogen.

In the subsequent sweeps, Fig. $8(\cdots)$ and $(\cdot \cdot)$, using a restricted potential range from $-0.40 \mathrm{~V}$ to $+1.40 \mathrm{~V}$, the voltammograms recorded showed the formation of the oxide layer and their reduction, although with very low peak currents.

In another experiment, after the application of the electrodeposition potential and transfer to the supporting electrolyte $\mathrm{pH} 4.5$, the first SW voltammogram recorded at $15 \mathrm{mVs}^{-1}$ showed the presence of a reversible oxidation peak at $+1.20 \mathrm{~V}$ with a very low current, Fig. 7C-D, thus confirming the electrodeposition of palladium oxide from the solutions of the Pd(II)-Spm and Pd(II)-Spd complexes.

The AFM and voltammetric results showed that application of high negative potentials during the sweeps in solution, Fig. 7A-B, or of a positive applied potential, Figs. 4D, 5D and 8, lead to the Pd(II)-Spm 
A
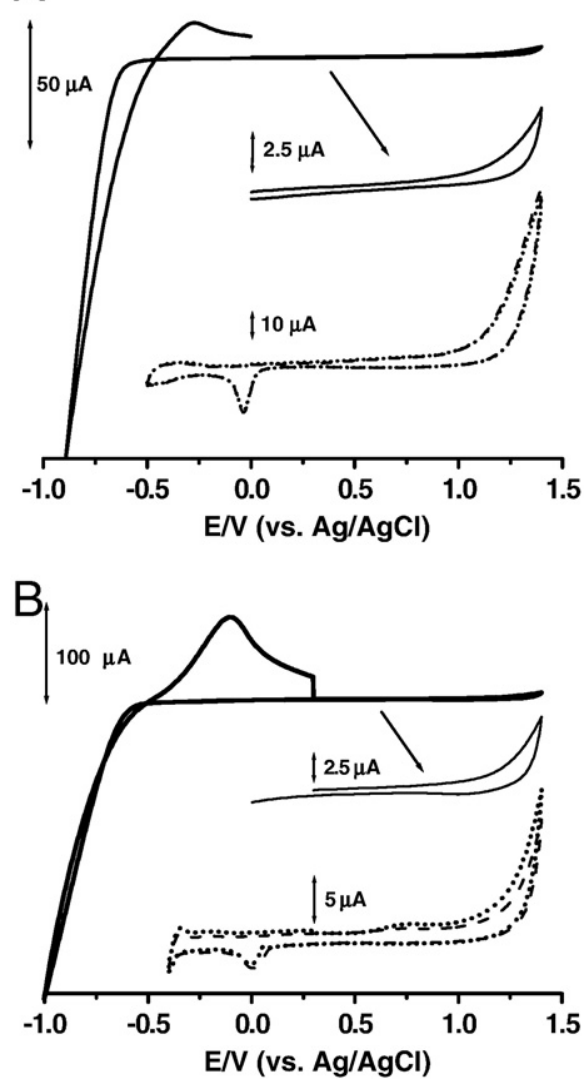

Fig. 8. CVs in pH 4.5 0.1 M acetate buffer of (A) Pd(II)-Spm and (B) Pd(II)-Spd, after electrodeposition at $+1.20 \mathrm{~V}$, vs. $\mathrm{Ag} / \mathrm{AgCl}$, during $30 \mathrm{~min}$ from $2 \mathrm{mM}$ solutions in $\mathrm{pH} 7.0$

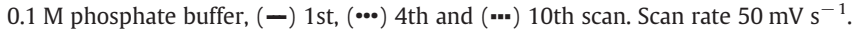

and $\mathrm{Pd}(\mathrm{II})-$ Spd dissociation and the deposition of palladium nanostructures on the surface of the HOPG and GC electrodes.

\section{Conclusions}

The redox behaviour of two polynuclear Pd(II) complexes with biogenic polyamines Spm and Spd, with great potential clinical importance due to their antiproliferative and cytotoxic activity coupled to less severe side-effects, was studied at room temperature, using AFM and voltammetry.

The ligands Spm and Spd, as well as the corresponding complexes $\mathrm{Pd}(\mathrm{II})-\mathrm{Spm}$ and $\mathrm{Pd}(\mathrm{II})-$ Spd present different adsorption patterns and degree of HOPG surface coverage, depending on the chemical structures, the dimensions, the solution concentration and the applied potential. The voltammetric study of Spm and Spd showed that these biogenic polyamines suffer oxidation at the GC electrode. The oxidation process is irreversible and dependent on $\mathrm{pH}$. In acid medium the polyamines are fully protonated and hardly suffer oxidation.

The Pd(II)-Spd complex has a stronger adsorption on HOPG and always presents larger peak currents at the GC electrode, due to the molecular structure incorporating three palladium atoms and two Spd molecules with greater adsorption, in comparison with $\mathrm{Pd}(\mathrm{II})-\mathrm{Spm}$ that has only two palladium atoms and one Spm molecule.

Applying high positive potentials, the stability of Pd(II)-Spm and Pd(II)-Spd complexes was tested. The voltammetric and AFM results show that palladium removal is possible for both $\mathrm{Pd}(\mathrm{II})-\mathrm{Spm}$ and Pd(II)-Spd, by applying either a high negative potential during the cycles in solution, or a fixed positive potential that leads to the formation of a mixed multilayer of palladium oxide, Spm/Spd and $\mathrm{Pd}(\mathrm{II})-\mathrm{Spm} / \mathrm{Pd}(\mathrm{II})-\mathrm{Spd}$ complexes on the carbon electrode surface.

\section{Acknowledgements}

Financial support from Fundação para a Ciência e Tecnologia (FCT), Post-Doctoral Grant SFRH/BPD/27087/2006 (A.-M. Chiorcea-Paquim), Ph.D. Grants SFRH/BD/18914/2004 (O. Corduneanu) and SFRH/BD/ 17493/2004 (S.M. Fiuza), project PTDC/QUI/65255/2006, POCI (cofinanced by the European Community Fund FEDER), and CEMUC-R (Research Unit 285), is gratefully acknowledged.

\section{References}

[1] F.A. Carey, Organic Chemistry, McGraw-Hill, Inc., New York, 2003, pp. 913-970.

[2] D.E. Metzler, Biochemistry, Elsevier Academic Press, 20031380 pp.

[3] A. Toninello, P. Pietrangeli, U. De Marchi, M. Salvi, B. Mondovi, Amine oxidases in apoptosis and cancer, BBA - Rev. Cancer 1765 (2006) 1-13.

[4] N. Seiler, J.G. Delcros, J.P. Moulinoux, Polyamine transport in mammalian cells. An update, Int. J. Biochem. Cell Biol. 28 (1996) 843-861.

[5] A.K. Abraham, A. Pihl, Role of polyamines in macromolecular synthesis, Trends Biochem. Sci. 6 (1981) 106-107.

[6] H. Deng, V.A. Bloomfield, J.M. Benevides, G.J. Thomas Jr., Structural basis of polyamine-DNA recognition: spermidine and spermine interactions with genomic B-DNAs of different GC content probed by Raman spectroscopy, Nucleic Acids Res. 28 (2000) 3379-3385.

[7] C. Navarro-Ranninger, F. Zamora, I. López-Solera, J.R. Masaguer, J.M. Pérez, C. Alonso, S. Martínez-Carrera, Palladium(II) salt and complexes of spermidine with a sixmember chelate ring. Synthesis, characterization, and initial DNA-binding and antitumor studies, J. Inorg. Biochem. 46 (1992) 267-279.

[8] C. Navarro-Ranninger, F. Zamora, J.R. Masaguer, J.M. Pérez, V.M. González, C. Alonso, Palladium(II) compounds of putrescine and spermine. Synthesis, characterization, and DNA-binding and antitumor properties, J. Inorg. Biochem. 52 (1993) 37-49.

[9] C. Navarro-Ranninger, P. Amo Ochoa, J.R. Masaguer, J.M. Pérez, V.M. González, C. Alonso, Platinum (II) and (IV) spermidine complexes. Synthesis, characterization, and biological studies, J. Inorg. Biochem. 53 (1994) 177-190.

[10] G. Codina, A. Caubet, C. López, V. Moreno, E. Molins, Palladium(II) and platinum(II) polyamine complexes, Helv. Chim. Acta 82 (1999) 1025-1037.

[11] M.P.M. Marques, T. Girão, M.C. Pedroso De Lima, A. Gameiro, E. Pereira, P. Garcia, Cytotoxic effects of metal complexes of biogenic polyamines. I. Platinum(II) spermidine compounds: prediction of their antitumour activity, BBA - Mol. Cell Res. 1589 (2002) 63-70.

[12] N. Farrell, Polynuclear Platinum Drugs, in: A. Sigel, H. Sigel (Eds.), Metal Complexes in Tumor Diagnosis and as Anticancer Agents, vol. 42 of Metal Ions in Biological Systems, Marcel Dekker Inc., New York, 1996, pp. 251-296.

[13] L.J. Teixeira, M. Seabra, E. Reis, M.T. Girão da Cruz, M.C. Pedroso de Lima, E. Pereira, M.A Miranda, M.P.M. Marques, Cytotoxic activity of metal complexes of biogenic polyamines: polynuclear Platinum(II) chelates, J. Med. Chem. 47 (2004) 2917-2925.

[14] S.M. Fiuza, A.M. Amado, P.J. Oliveira, V.A. Sardão, L.A.E. Batista de Carvalho, M.P.M. Marques, Pt(II) vs Pd(II) polyamine complexes as new anticancer drugs: a structure-activity study, Lett. Drug Des. Discov. 3 (2006) 149-151.

[15] B. Moriarity, O. Nováková, N. Farrell, V. Brabec, J. Kašpárková, 1, 2-GG intrastrand cross-link of antitumor dinuclear bifunctional platinum compound with spermidine linker inhibits DNA polymerization more effectively than the cross-link of conventional cisplatin, Arch. Biochem. Biophys. 459 (2007) 264-272.

[16] T. Gebel, H. Lantzsch, K. Pleßow, H. Dunkelberg, Genotoxicity of platinum and palladium compounds in human and bacterial cells, Mutat. Res., Genet. Toxicol. Environ. Mutagen. 389 (1997) 183-190.

[17] R.D. Hubbard, S. Fidanze, Alkylating and platinum antitumor compounds, Comprehensive Medicinal Chemistry II, vol. 7, 2007, pp. 129-148.

[18] S. Aebi, B. Kurdi-Haidar, R. Gordon, B. Cenni, H. Zheng, D. Fink, R.D. Christen, C.R Boland, M. Koi, R. Fishel, S.B. Howell, Loss of DNA mismatch repair in acquired resistance to cisplatin, Cancer Res. 56 (1996) 3087-3090.

[19] R.D. Graham, D.R. Williams, The synthesis and screening for anti-bacterial, -cancer -fungicidal and -viral activities of some complexes of palladium and nickel, J. Inorg. Nucl. Chem. 41 (1979) 1245-1249.

[20] M.M.L. Fiallo, A. Garnier-Suillerot, Interaction of the antitumor drug streptonigrin with palladium(II) ions. Evidence of the formation of a superoxo-palladium(II)streptonigrin, Inorg. Chem. 29 (1990) 893-897.

[21] A.S. Soares, S.M. Fiúza, M.J. Gonçalves, L.A.E.B. de Carvalho, M.P.M. Marques, A.M. Urbano, Effect of the metal center on the antitumor activity of the analogous dinuclear spermine chelates $\left(\mathrm{PdCl}_{2}\right)_{2}$ (spermine) and $\left(\mathrm{PtCl}_{2}\right)_{2}$ (spermine), Lett. Drug Des. Discov. 4 (2007) 460-463.

[22] A. Garoufis, S.K. Hadjikakou, N. Hadjiliadis, Palladium coordination compounds as anti-viral, anti-fungal, anti-microbial and anti-tumor agents, Coord. Chem. Rev. 253 (2009) 1384-1397.

[23] M. Zeizinger, J.V. Burda, J. Šponer, V. Kapsa, J. Leszczynski, A systematic ab initio study of the hydration of selected palladium square-planar complexes. A comparison with platinum analogues, J. Phys. Chem., A 105 (2001) 8086-8092.

[24] A.S. Abu-Surrah, H.H. Al-Sadoni, M.Y. Abdalla, Palladium-based chemotherapeutic agents: routes toward complexes with good antitumor activity, Cancer Ther. 6 (2008) $1-10$.

[25] A.S. Abu-Surrah, M. Kettunen, K. Lappalainen, U. Piironen, M. Klinga, M. Leskelä, Synthesis of new chiral diimine palladium(II) and nickel(II) complexes bearing oxazoline- and myrtanyl-based nitrogen ligands. Crystal structure of the C2-symmetric complex [\{(1R,2S)-inda-box\}PdCl2], Polyhedron 21 (2002) 27-31. 
[26] A.I. Anzellotti, M. Sabat, N. Farrell, Covalent and noncovalent interactions for [Metal(dien)nucleobase] ${ }^{2+}$ complexes with l-tryptophan derivatives: formation of palladium-tryptophan species by nucleobase substitution under biologically relevant conditions, Inorg. Chem. 45 (2006) 1638-1645.

[27] A.M. Amado, S.M. Fiuza, M.P.M. Marques, L.A.E. Batista de Carvalho, Conformational and vibrational study of platinum(II) anticancer drugs: cis-diamminedichloroplatinum(II) as a case study, J. Chem. Phys. 127 (2007) 185104-185113.

[28] S.M. Fiuza, A.M. Amado, M.P.M. Marques, L.A.E. Batista de Carvalho, Use of effective core potential calculations for the conformational and vibrational study of Platinum(II) anticancer drugs. cis-diamminedichloroplatinum(II) as a case study, J. Phys. Chem., A 112 (2008) 3253-3259.
[29] M.A. Witek, G.M. Swain, Aliphatic polyamine oxidation response variability and stability at boron-doped diamond thin-film electrodes as studied by flow-injection analysis, Anal. Chim. Acta 440 (2001) 119-129.

[30] O. Corduneanu, V.C. Diculescu, A.-M. Chiorcea Paquim, A.M. Oliveira Brett, Shapecontrolled palladium nanowires and nanoparticles electrodeposited on carbon electrodes, J. Electroanal. Chem. 624 (2008) 97-108.

[31] V.C. Diculescu, A.-M. Chiorcea Paquim, O. Corduneanu, A.M. Oliveira Brett, Palladium nanoparticles and nanowires deposited electrochemically: AFM and electrochemical characterization, J. Solid State Electrochem. 11 (2007) 887-898. 